\title{
Publisher's Note: Resilience of hidden order to symmetry-preserving disorder [Phys. Rev. B 96, 214206 (2017)]
}

Marcello Calvanese Strinati, Davide Rossini, Rosario Fazio, and Angelo Russomanno

(Q) (Received 2 January 2018; published 10 January 2018)

DOI: 10.1103/PhysRevB.97.019903

This paper was published online on 20 December 2017 with a typographical error on page 12. On page 12, right-hand column, the first sentence after Eq. (B5b) should read as "For the plots in Fig. 12, we choose $k=0.2 L, L=120$, as in Eq. (B1)." The paper has been corrected as of 27 December 2017. The text is correct in the printed version of the journal. 